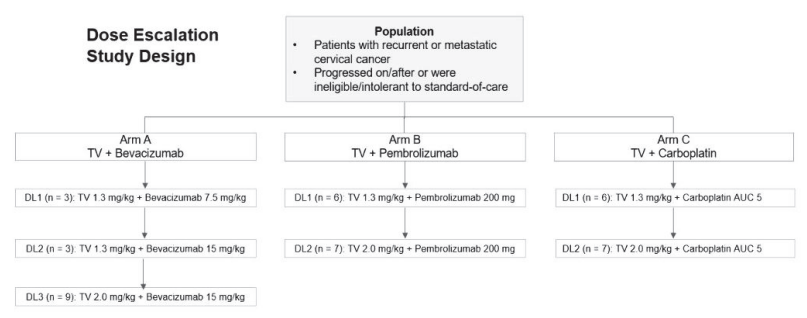

Abstract 0013/\#573 Figure 1

AUC, area under the curve; DL, dose level; DLT, dose-limiting toxicity; TV, tisotummab vedotin.

Drugs administered IV on day 1 of 21-day cycle.

Patients were treated for at least 2 cycles to evaluate DLTs.

Escalation to the next DL was permited when fewer than 1 in 3 patients at the current DL experienced a DLT.

The recommended phase 2 does (RP2D) was highest planned DL reached with DLTs in no more than 1 in 6 patients.

TV $2.0 \mathrm{mg} / \mathrm{kg}+$ pembrolizumab $200 \mathrm{mg}$ Q3W. Two patients had cOR. In Arm C, 13 patients received 5 cycles (median) of $\mathrm{TV}+4$ cycles of carboplatin. Grade $\geq 3$ AEs occurred in 8 patients (62\%). The RP2D was TV $2.0 \mathrm{mg} / \mathrm{kg}+$ carboplatin AUC 5 Q3W. Four patients had cOR.

Conclusions TV plus bevacizumab, pembrolizumab, or carboplatin was tolerated and had an acceptable safety profile with encouraging activity in patients with $\mathrm{r} / \mathrm{mCC}$.

\section{4/\#828 EFFICACY AND SAFETY OF SOCAZOLIMAB IN RECURRENT OR METASTATIC CERVICAL CANCER: RESULTS FROM THE PHASE I TRIAL FOLLOWED BY AN EXPANSION STUDY}

${ }^{1} \mathrm{~J} \mathrm{An}{ }^{*}$, ${ }^{2} \mathrm{~J}$ Tang, ${ }^{3} \mathrm{H}$ Xiong, ${ }^{4} \mathrm{H}$ Qiu, ${ }^{4} \mathrm{~L} \mathrm{Luo},{ }^{5} \mathrm{~L}$ Wang, ${ }^{6} \mathrm{D}$ Wang, ${ }^{7} \mathrm{Q}$ Zhou, ${ }^{8} \mathrm{Q} \mathrm{Xu},{ }^{9} \mathrm{H}$ Song, ${ }^{10} \mathrm{Y}$ Zhang, ${ }^{11} \mathrm{H}$ Zhang, ${ }^{3} \mathrm{G} \mathrm{Li},{ }^{1} \mathrm{~L}$ Wu. ${ }^{1}$ Cancer Hospital Chinese Academy of Medical Science, Oncology, Beijing, China; ${ }^{2}$ Hunan Province Cancer Hospital, Oncology, Changsha, China; ${ }^{3}$ Wuhan Tongji Hospital, Oncology, Wuhan, China; ${ }^{4}$ Wuhan University Zhongnan Hospital, Oncology, Wuhan, China; ${ }^{5}$ Henan Province Cancer Hospital, Oncology, Zhengzhou, China; 'Liaoning Province Cancer Hospital, Oncology, Shenyang, China; ${ }^{7}$ Chongqing Cancer Hospital, Oncology, Wuhan, China; ${ }^{8}$ Fujian Province Cancer Hospital, Oncology, Fuzhou, China; ${ }^{9}$ Guangxi Medical University Affiliated Tumor Hospital, Oncology, Nanning, China; ${ }^{10}$ Haerbin Medical University Affiliated Tumor Hospital, Oncology, Haerbin, China; ${ }^{11}$ Yunnan Province Tumor Hospital, Oncology, Kunming, China

\subsection{6/ijgc-2021-IGCS.14}

Objectives This study (ClinicalTrials.gov identifier: NCT03676959) is a phase I plus expansion study investigating the antitumor activity and safety of socazolimab, an anti-PDL1 monoclonal antibody, in recurrent or metastatic cervical cancer.

Methods All patients received one or more lines of chemotherapy for recurrent or metastatic disease. Patients received $5 \mathrm{mg} /$ $\mathrm{kg}$ socazolimab every 2 weeks as one cycle for total of 24 cycles. Tumor imaging was performed every 8 weeks for 48 weeks. The primary end points were safety and objective response rate (ORR) assessed per RECIST 1.1 evaluated by independent radiology review committee (IRC). Progression free survival (PFS), duration of response (DOR) and overall survival (OS) were the secondary end points.

Results Ninety-four patients were treated. Median age was 51.5 years, and $58.5 \%$ of patients had ECOG performance status of 1. Fifty-six patients had PD-L1-positive tumors
(CPS $\geq 1 \%$ ). Twenty-nine patients had PD-L1-negative tumors $(\mathrm{CPS}<1)$. Median follow-up was 14.75 months. ORR was $18.1 \%$ (95\% CI, $10.9 \%$ to $27.4 \%$ ), with 3 complete and 14 partial responses. PD-L1-positive patients had an ORR of $19.6 \%$ (95\% CI, $10.2 \%$ to $32.4 \%$ ). PD-L1-negative patients had an ORR of $20.7 \%$ (95\% CI, $8.0 \%$ to 39.7\%). Median PFS and OS were 4.5 and 15.8 months respectively. Median DOR was not reached. TRAE occurred in $62.1 \%$ of patients. Grade 3 to 4 TRAE occurred in $8.4 \%$ of patients.

Conclusions Socazolimab demonstrated durable antitumor activity and manageable safety in patients with recurrent or metastatic cervical cancer. Based on these results, China National Medical Products Administration (NMPA) grants breakthrough Therapy Designation to socazolimab for the cervical cancer indication.

\section{Plenary 6}

\section{5/\#436 COMPREHENSIVE GENOMIC PROFILING OF LOW- GRADE SEROUS CANCERS AND ASSOCIATED CLINICAL OUTCOMES}

${ }^{1}$ B Manning-Geist*, 'S Gordhandas, 'A Da Cruz Paula, 'B Weigelt, ${ }^{2} Y$ Liu, ${ }^{3} \mathrm{H}$ Chui, ${ }^{2} \mathrm{R}$ Grisham. 'Memorial Sloan Kettering Cancer Center, Gynecology Service, Department of Surgery, New York, USA; ${ }^{2}$ Memorial Sloan Kettering Cancer Center, Medicine, New York, USA; ${ }^{3}$ Memorial Sloan Kettering Cancer Center, Pathology, New York, USA

\subsection{6/ijgc-2021-IGCS. 15}

Objectives The genetic landscape of low-grade serous carcinomas (LGSCs) is poorly described. We sought to characterize somatic mutational profiles of LGSCs and correlate findings with clinical outcomes.

Methods Patients with LGSC $(n=123)$ consented to an IRBapproved protocol of tumor-normal massively parallel sequencing that included 341-505 cancer-related genes. Clinical outcomes and frequency of genetic alterations, including somatic mutations and copy number alterations, were assessed and compared using the Fisher exact test.

Results Among 123 sequenced tumors, median tumor mutational burden was low (1.8 mutations/Mb). Recurrent alterations included those affecting KRAS (32\%), NRAS (11\%), BRAF (10\%), CDKN2A (9\%), and ERBB2 (5\%) (see figure 1). KRAS mutations were more frequent in patients $\geq 50$ years of age at LGSC diagnosis than in patients $<50$ years of age ( $41 \%$ vs $23 \%$, respectively; $\mathrm{p}<0.05$ ); in those with platinumsensitive vs. platinum-resistant disease ( $41 \%$ vs $19 \%$, respectively; $\mathrm{p}=0.05)$; and in patients surviving $\geq 5$ years compared with $<5$ years $(39 \%$ vs $11 \%$, respectively; $\mathrm{p}<0.05)$. BRAF mutations were more frequent in platinum-resistant compared with platinum-sensitive disease $(19 \%$ vs $3 \%$, respectively; $\mathrm{p}<0.05)$. CDKN2A alterations were associated with risk of recurrence, while none of the patients with CDKN2A-wildtype tumors developed recurrence $(13 \%$ vs $0 \%$, respectively; $\mathrm{p}<0.05)$. Recurrence was also associated with higher median fraction of genome altered $(\mathrm{p}<0.05)$.

Conclusions The presence of KRAS mutations in LGSC may be associated with less aggressive phenotypes and older age at diagnosis. CDKN2A and BRAF alterations may be associated with recurrence and platinum resistance, respectively. 
No recurrences $(n=27)$

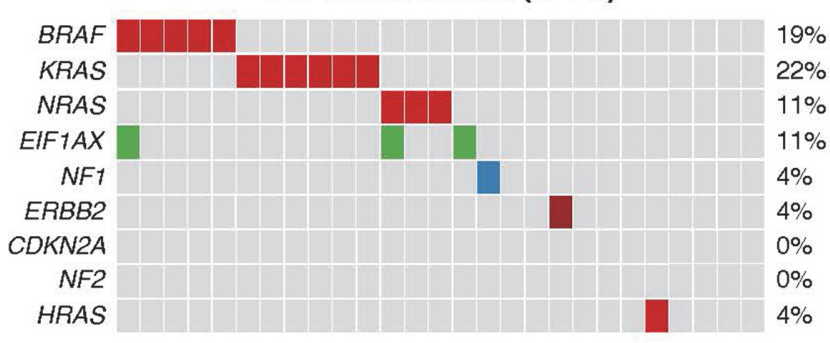

Platinum sensitive $(n=68)$
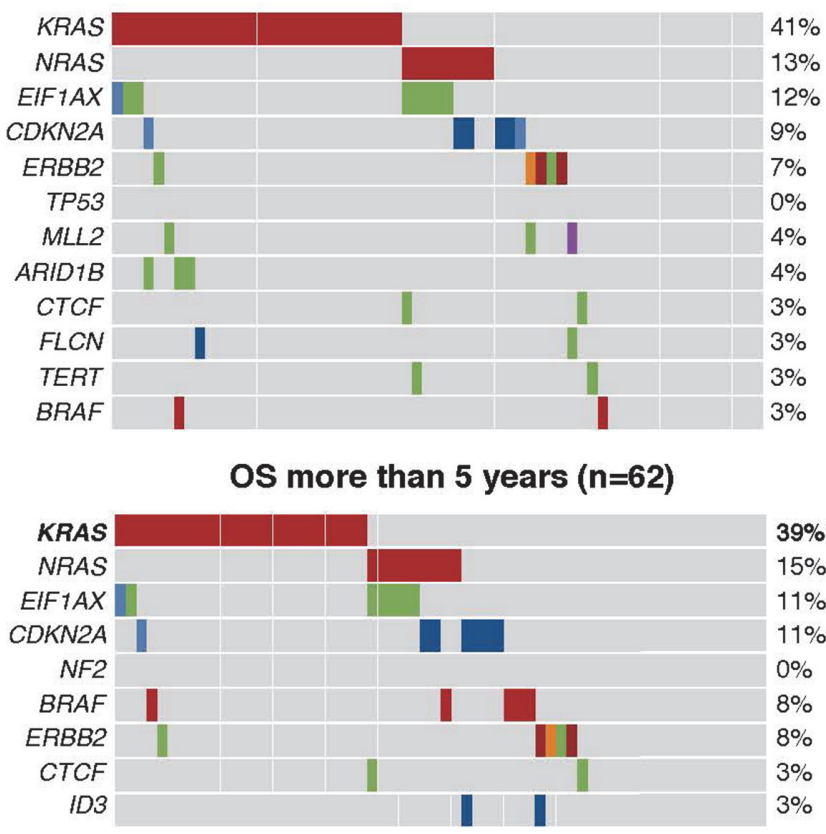

Less than 50 years $(n=65)$

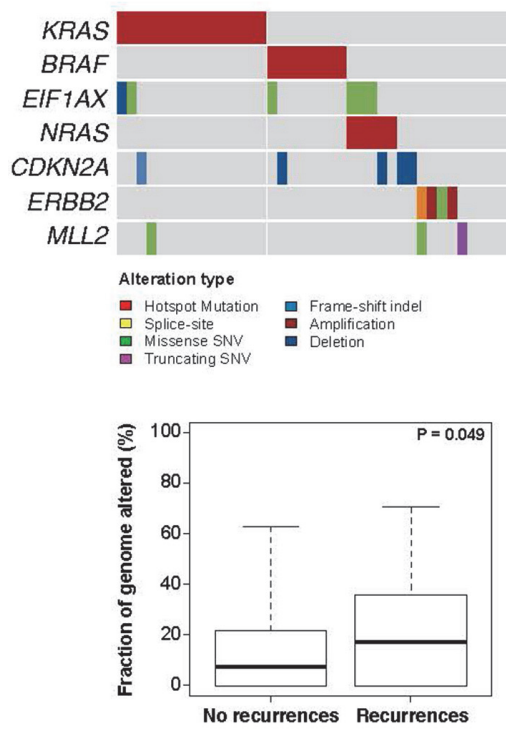

Abstract 0015/\#436 Figure 1
Recurrences ( $n=96$ )

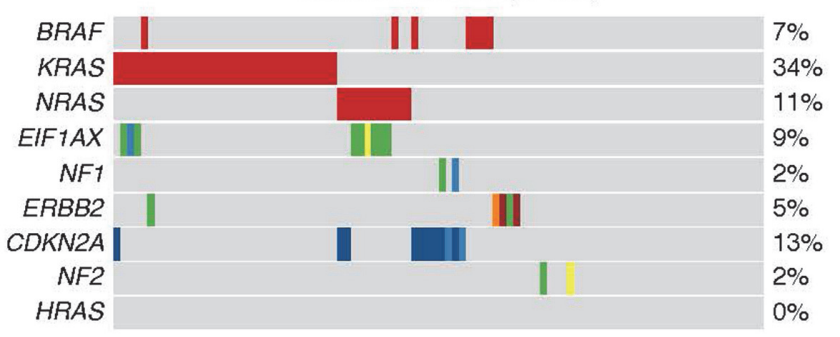

Platinum resistant $(\mathrm{n}=27)$

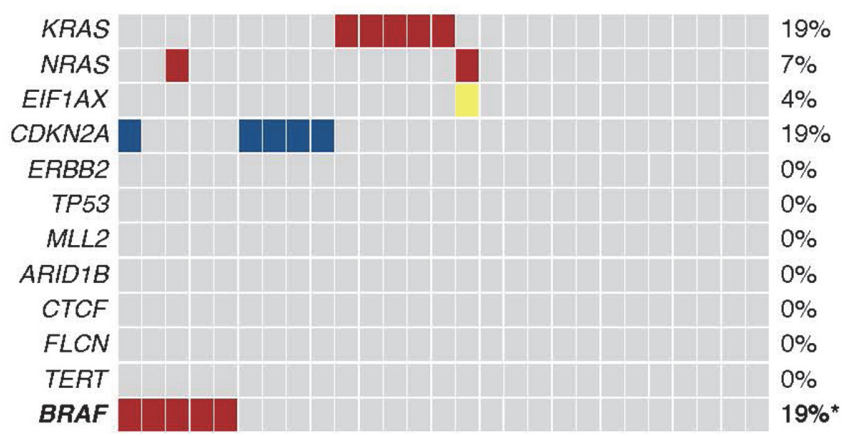

OS less than 5 years $(n=19)$

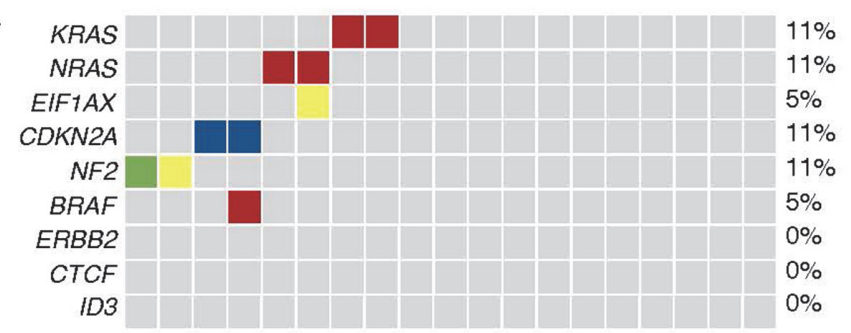

More than 50 years $(n=58)$

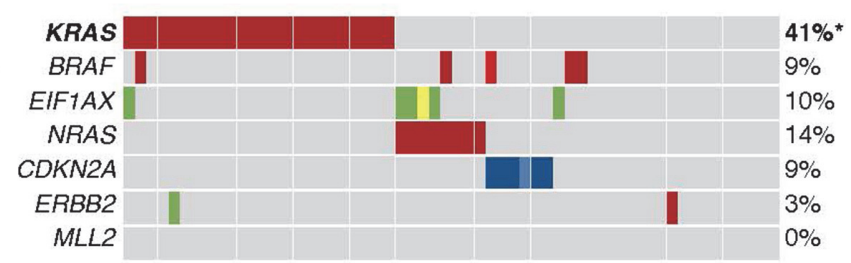

